\title{
Artículos
}

\section{Enseñanza de lenguas: una comparación de las representaciones sociales de alumnos de primer y quinto año de la carrera de Lingüística Aplicada a la Traducción}

Ensino de línguas: uma comparação das representações sociais de alunos do primeiro e quinto anos da carreira de Lingüística Aplicada à Tradução

Language teaching: a comparison of social representations held by first- and fifth-year students of the Translation Studies undergraduate programme at Universidad de Santiago de Chile

\author{
Néstor Singer ${ }^{1}$ \\ Manuel Rubio ${ }^{2}$ \\ Raquel Rubio ${ }^{3}$
}

1. Profesor Asociado. Departamento de Lingüística y Literatura. Facultad de Humanidades. Universidad de Santiago de Chile. Santiago, Chile. http://orcid.org/0000-0001-7772-9906. E-mail: nestor.singer@usach.cl.

2. Profesor Asociado. Departamento de Lingüística y Literatura. Facultad de Humanidades. Universidad de Santiago de Chile. Santiago, Chile. https://orcid.org/0000-0003-2314-1781. E-mail: manuel.rubio@usach.cl.

3. Profesor Asociado. Departamento de Lingüística y Literatura. Facultad de Humanidades. Universidad de Santiago de Chile. Santiago, Chile. https://orcid.org/0000-0003-4997-3543. E-mail: raquel.rubio@usach.cl. 


\section{RESUMEN}

Este artículo compara las representaciones sobre el proceso de enseñanza y aprendizaje de lenguas extranjeras que tienen estudiantes de primer y quinto año de la carrera de Lingüística Aplicada a la Traducción de la Universidad de Santiago de Chile (USACH). El concepto de representación social se toma desde la psicología social (Jodelet 1988, 2008; Moscovici, 1979). Se utilizó una asociación libre de conceptos y una entrevista semiestructurada a cuatro grupos de estudiantes. Los resultados reflejan cambios significativos relacionados con el dominio, enseñanza y aprendizaje de las lenguas estudiadas. Se concluye que dichos cambios facilitarían la adopción de nuevas metodologías para los cursos de lenguas extranjeras en este programa de traducción.

Palabras clave: representaciones sociales; estudios de traducción; enseñanza de segundas lenguas; formación de traductores.

\section{RESUMO}

O artigo compara as representações sobre o processo de ensinoaprendizagem de línguas estrangeiras que estudam os alunos do primeiro e quinto anos da graduação em Linguística Aplicada à Tradução da Universidad de Santiago de Chile (USACH). Tem como aparato teórico a representação social na perspectiva da psicologia social (Jodelet 1988 , 2008; Moscovici 1979). Utilizou-se uma associação livre de conceitos e uma entrevista semi-estruturada a quatro grupos de estudantes. Os resultados refletem mudanças significativas com o domínio, o ensino e a aprendizagem das línguas estudadas. Conclui-se que tais mudanças contribuiriam à adoção de novas metodologias para os cursos de línguas estrangeiras no programa de tradução.

Palavras-chave: representações sociais; estudos de tradução; ensino de segundas línguas; formação de tradutores.

\footnotetext{
ABSTRACT

This article compares the social representations about the processes of teaching and learning a foreign language held by first-and fifth-year students of the translation studies undergraduate programme at Universidad de Santiago de Chile (USACH). The concept of social representations is taken from social psychology (Jodelet 1988, 2008; Moscovici 1979).A
} 
word association task and a semi structured interview were administered to four groups of translator trainees. Results show significant changes in terms of language proficiency, teaching and learning. It is concluded that such changes would promote the adoption of new methodologies for the foreign language course units in this translation programme.

Keywords: social representations; translation studies; second language teaching; translator education.

\section{Introducción}

La enseñanza de la traducción a nivel de pregrado ha sido un tema que ha recibido particular atención en los últimos años. Durante la década de los años 90, se pudo apreciar la proliferación de modelos que daban cuenta de la competencia traductora (Hurtado, 2017), entendida como el conocimiento teórico y práctico necesario para traducir. Los programas de formación de traductores a nivel de pregrado han utilizado estos modelos como referencia para diseñar su currículum y asignaturas. Esto ha generado una serie de posibles enfoques y metodologías para la enseñanza de la traducción, donde destacan los trabajos de Hurtado (1999), Kelly (2005), Kiraly (2000), entre otros. Dichos trabajos suponen el aprendizaje significativo y profundo mediante un enfoque constructivista.

En el caso de Latino América, la literatura presenta numerosos casos de sistematizaciones de dichas estrategias aplicadas (véase Calvo, 2017, Morales, 2017, Carvajal, 2017) particularmente en contextos monolingües, donde los programas de pregrado apuntan a desarrollar las habilidades para traducir, así como también la competencia comunicativa en las lenguas de trabajo. No obstante, tal como señala Cruz García (2017), es justamente en este último punto, es decir la enseñanza de lenguas en contexto de la formación de traductores, en que no se ha avanzado significativamente. Esto porque, si bien se ha planteado la enseñanza de lenguas para estudiantes de traducción, dichas propuestas metodológicas no han sido mayormente adoptadas en los cursos de lengua en los programas de formación de traductores. En este sentido, destacan los enfoques basados en tipos textuales (Beeby, 2004) o corpus (Bernardini, 2004b, 2016), que apuntan a un desarrollo integral de la lengua y al fomento de la competencia traductora de los 
estudiantes. Ambas metodologías se caracterizan por el alto nivel de autogestión del aprendizaje por parte de los alumnos y un rol activo en su proceso de formativo.

Entre las posibles dificultades para la inclusión de estas metodologías, se pueden destacar dos factores críticos. Por una parte, el perfil de los profesores de lengua en estos contextos es difuso. A la fecha, no existen estudios que establezcan qué hacen los profesores de lengua en sus clases dirigida a futuros traductores y las metodologías que emplean en este contexto. Por otra parte, existe la complejidad asociada al individuo que estudia traducción, pues estos enfoques parecieran diferir de los constructos tradicionales de educación experimentados por los alumnos durante su etapa escolar: pasividad, discontinuidad del contenido y falta de instancias de práctica significativa. Por lo tanto, las representaciones que portan en cuanto a los procesos de enseñanzaaprendizaje de una lengua extranjera, así como motivaciones intrínsecas y extrínsecas pueden ser factores capaces de facilitar o complejizar la adopción de nuevas metodologías y estrategias en la clase de lengua extranjera.

Dichas representaciones corresponden a un conocimiento de sentido común construido en la interacción con otros. Este conocimiento configura la subjetividad con que un individuo concibe y participa en determinadas prácticas sociales (Jodelet, 1988, 2008; Moscovici, 1979; Kornblit, 2007). Desde esta perspectiva, indagar en las representaciones sociales que comparten los estudiantes de traducción respecto del aprendizaje de una lengua extranjera, apunta a establecer las formas de pensar y sentir que han ido conformando en el proceso de su adquisición y uso en diversos espacios de interacción, sean estos presenciales o virtuales. Obviamente, con la experiencia, dichas representaciones pueden ir cambiando y se constituyen en un factor clave que condiciona el despliegue del compromiso y habilidades para el aprendizaje de una lengua.

En la línea de avanzar en la formulación de planteamientos teóricos que orienten nuevas propuestas en el diseño curricular de programas de enseñanza de lenguas en la formación de traductores en contextos monolingües, interesa comparar las representaciones sobre el proceso de enseñanza y aprendizaje de una lengua extranjera que portan los 
estudiantes que inician y los que están finalizando su carrera profesional como traductores. Para hacerlo, se diseñó un estudio cualitativo de caso único que considera el discurso de los estudiantes como la unidad de análisis. En otras palabras, la labor interpretativa configurada tiene como límite explicitar los elementos manifiestos y subyacentes del discurso de los estudiantes.

Congruentemente con lo anterior, el propósito de este artículo es comparar las representaciones expresadas por estudiantes de primero y quinto año de la carrera de Lingüística Aplicada a la Traducción sobre la enseñanza y aprendizaje de lenguas extranjeras. Es importante señalar que este trabajo no ahonda las representaciones sobre la labor traductora, pues el objetivo es determinar si las representaciones sobre el aprendizaje y enseñanza de lenguas extranjeras permitirían la inclusión de nuevas propuestas metodológicas al respecto.

\section{Marco teórico}

\section{Competencia traductora y la enseñanza de lenguas en la formación de pregrado}

La lingüística aplicada, la psicología y la pedagogía han tenido un gran impacto en la comprensión de la traducción y, por tanto, en la conceptualización de la competencia traductora (Hurtado, 2017). En concreto, desde los años 70 se han postulado una serie de modelos sobre la competencia traductora informados tanto por los avances y tendencias dentro de las miradas críticas de la traducción, así como resultado de momentos históricos y sociales coyunturales.

Así, desde una perspectiva histórica, podemos apreciar una serie de modelos con nomenclatura similar en búsqueda de aquel que pudiese definir de mejor manera la competencia traductora. Las discusiones en torno a esta materia han tenido su auge durante la década de los años 90. Al observar dichas propuestas (Wills, 1976; Delisle, 1980; Roberts, 1984; Hewson y Martin, 1991; Nord, 1991, 1992; Neubert, 1994; Kiraly, 1995; Hurtado, 1996a, 1996b; Hansen, 1997; Risku, 1998), se puede observar que la problemática se ha centrado en el proceso de traducción y no en la competencia lingüística y comunicativa necesaria 
para poder traducir. Más aún, se evidencia que en todas las propuestas de los modelos se hace alusión a dicha competencia comunicativa en lenguas extranjeras como un supuesto dado. En concreto, si se observa el modelo del PACTE (2017) denominado Proceso de Adquisición de Competencia Traductora y Evaluación, la competencia traductora se perfila en cinco sub-competencias: bilingüe, extralingüística (conocimiento bicultural, enciclopédico y específico), conocimiento sobre traducción (conocimiento declarativo y procedimental para traducir), instrumental (manejo de herramientas de traducción) y estratégica (toma de decisiones conscientes e inconscientes para resolver problemas de traducción). Además, el modelo supone la existencia de componentes psicofisiológicos (recursos actitudinales, cognitivos y psicomotores). En definitiva, presupone una competencia lingüística y comunicativa en las lenguas extranjeras de trabajo, lo cual es relevante en países monolingües como Chile, donde los conocimientos previos de los estudiantes sobre lenguas extranjeras son escasos. En el caso particular de Lingüística Aplicada a la Traducción, los alumnos tienen el español como lengua materna (lengua A) y trabajan con inglés y portugués como lenguas $\mathrm{B}$, es decir, traducen desde y hacia esas lenguas, y japonés como lengua $\mathrm{C}$, esto es, traducen solo desde esa lengua hacia el español.

En este sentido, los programas de traducción a nivel de pregrado debiesen apuntar a la enseñanza de lenguas junto con el desarrollo de la competencia traductora tal como ha sido definida anteriormente. Bernardini (2000) considera tres principios clave en la formación de traductores: conciencia, reflexión e ingenio (traducción propia). El primero se relaciona con el pensamiento crítico hacia el texto a traducir, considerando no solo su contenido sino la forma en que está expresando. Esto forma parte fundamental de la actitud ética y profesional del trabajo del traductor. El segundo tiene que ver con la capacidad de practicar y forma de utilizar estrategias específicas en los procesos de traducción. El último principio se relaciona con el desarrollo de la capacidad de utilizar autónomamente recursos finitos para resolver problemas de traducción no finitos. En síntesis, se aprecia que estos principios apuntan al proceso de desarrollo de la competencia traductora y no a la condición de base: el desarrollo de una competencia comunicativa en una lengua extranjera sin la cual la conciencia, la reflexión y el ingenio se ven obstaculizados. 
En efecto, la falta de dominio de las lenguas implicadas en el proceso de traducción llama al eclecticismo metodológico, pues, tal como señala Wilss (2004:13), “...puede que no exista un solo enfoque en la enseñanza de la traducción que, incluso teóricamente, sea capaz de organizar [la] vasta pluralidad de preocupaciones y especializaciones" (traducción propia). Indudablemente, esto supone que traducción y aprendizaje de lengua en contextos monolingües interactúen de manera permanente. Respecto de la competencia traductora propiamente tal, la literatura evidencia una serie de estrategias para abordar la enseñanza de traducción en pregrado. Por ejemplo, Agost (2008) apunta a cambios metodológicos en una de sus cátedras de iniciación a la traducción, mientras que Cortés (2009) narra las implicaciones de encargos de traducción para fomentar el aprendizaje significativo para preparar a sus alumnos de mejor forma para el mundo laboral, especialmente si se considera que una de las mayores necesidades del mercado a nivel internacional corresponde a la traducción especializada. Sin embargo, Wilss (2004) advierte que lo ideal es que los programas de pregrado permitan a los alumnos conocer la mayor cantidad de campos para prevenir la sobrespecialización. En esta misma línea, Bernardini (2004a) sugiere que el currículum del pregrado y las metodologías consideren las necesidades lingüísticas profesionales de los traductores e incluyan como eje los tres principios de conciencia, reflexión e ingenio, ya que estos serán útiles independientemente el nicho de mercado donde ejerzan los futuros traductores.

Esto último genera ciertas preguntas en cuanto a cuáles serían esas necesidades y, particularmente, aquellas relacionadas con el aprendizaje de lenguas extranjeras dentro de los programas de pregrado en traducción. La Bundesverband der Dolmetscher und Übersetzer (BDÜ - Asociación Nacional Alemana de Traductores e Intérpretes), en Kiraly (2000:164), señala que dicho conocimiento lingüístico se refiere a la habilidad de usar adecuadamente la lengua en cuanto a sus dimensiones pragmático-contextuales y temáticas, así como la capacidad metacognitiva de reflexionar y analizar dicha lengua.

Lo anterior plantea el desafío de cómo desarrollar dicha competencia en los estudiantes de traducción en contextos monolingües, pues dichos programas abordan tanto la enseñanza de lenguas $\mathrm{B}$ y $\mathrm{C}$, así como el desarrollo de habilidades traductoras. 


\section{Enseñanza de lenguas en estudios de traducción}

En los últimos años, la enseñanza de lengua en el contexto de la formación profesional de traductores ha recibido una mayor atención de la que se le asignaba hace años atrás. Las primeras reflexiones en torno a la enseñanza de idiomas para traductores provienen de la problematización de la traductología aplicada. En dicho contexto, Holmes (1972) aborda la naturaleza de los estudios de traducción e identifica tres dimensiones: política traductora, crítica traductora y la enseñanza de la traducción. En relación a esto último, analiza el rol que ha tenido la traducción como enfoque para la enseñanza de idiomas y cuestiona, entonces, el tipo de gramática que se necesitaría para enseñar idiomas dentro de un programa de formación de traductores. Años más tarde, Berenguer (1996) propone que esto correspondería a la enseñanza de lenguas con fines específicos. Dichos fines estarían establecidos dentro de la competencia traductora, que Kelly (2005:73) señala deberían centrarse en "...las habilidades lingüísticas que un traductor necesita” (traducción propia).

Según Berenguer (1996), la enseñanza de lenguas en estos programas debería: 1) potenciar la comprensión lectora, 2) aprender a disociar las lenguas en contacto, 3) familiarizar al estudiante con el uso de diccionarios y otros recursos, 4) transformar a los alumnos en expertos en cultura, y 5) sensibilizar al alumno en la actividad traductora. Adicionalmente, Hurtado (1999) señala el desarrollo de las habilidades de comprensión y producción escrita, desarrollar la oralidad, ampliar el bagaje sociocultural de los alumnos, familiarizar al estudiante con fuentes de documentación y, por último, desarrollar conocimiento lingüístico que se evidencie en el contraste de las lenguas de trabajo. Estos lineamientos apuntan a una clase de lengua que desarrolle la competencia lingüística, pero que además potencie el desarrollo temprano de otras competencias.

En esta línea, se han propuesto algunas alternativas interesantes de trabajo como los pre-sílabos basados en la traducción y orientados al estudiante, y los basados en el género discursivo orientados a la traducción (Beeby, 2004). Por su parte, Bernardini (2004b, 2016) propone el uso de corpus para propósitos de enseñanza de lenguas en traducción como alternativa que potencia el pensamiento crítico 
y autónomo de los alumnos, mientras que Singer (2016) sugiere una serie de principios para la adopción de este enfoque en las clases de lengua.

Si bien las propuestas mencionadas son válidas en sí mismas, presentan algunos desafíos para su ejecución en el aula en contextos monolingües como el chileno. Primero, suponen que el profesor de lengua es traductor o, a lo menos, con un gran conocimiento lingüístico de dos lenguas. Segundo, evidencian que el docente de lenguas en estudios de traducción también debe conocer lo que implica traducir y como su asignatura influye en dicho proceso. En otras palabras, debería estar al tanto de lo que es la competencia traductora. Por último, las propuestas suponen una orientación hacia el alumno debido al rol activo que cumplen y su futuro como traductores, pero no evidencian la inclusión del individuo (sus intereses, preocupaciones, conocimiento previo, bagaje cultural) como agente en sí en el proceso de formación traductora. Es esto último, lo que supone el mayor obstáculo para la adopción e implementación de dichas metodologías en el aula. Para poder integrar dicho repertorio metodológico a las clases de lenguas $\mathrm{B}$ y $\mathrm{C}$, el análisis de las representaciones sociales de los alumnos se perfila como una estrategia viable para establecer hasta que punto están dispuestos a gestionar su aprendizaje (Singer et al., 2018), así como determinar que entienden por una clase de lengua, los factores que promueven su éxito o fracaso. En consecuencia, este estudio se perfila como un primer acercamiento para determinar cómo los alumnos conciben el proceso de enseñanza aprendizaje de una lengua extranjera en el contexto de la formación de traductores. Los datos recogidos permitirán evaluar posteriormente la viabilidad para la implementación y adaptación de algunos de los enfoques señalados anteriormente.

\section{Representaciones sociales y enseñanza de lenguas}

El concepto de representación social surge en el ámbito de la psicología social y, gradualmente se ha introducido en el ámbito del estudio de los procesos de enseñanza y aprendizaje, fundamentalmente por la potencialidad que posee para explicar la construcción de un saber compartido que condiciona las prácticas de aprendizaje como un proceso activo y social (Chaib, 2015). Dicho concepto se enfoca en la 
importancia del conocimiento de sentido común construido a partir de diversas fuentes (formales, informales y no-formales), constituyéndose en un acervo cultural desde el cual tanto profesores como estudiantes concurren a la interacción didáctica, definen su rol y despliegan sus esfuerzos de enseñanza y aprendizaje.

Según Chaib (2015), un aspecto clave de todo proceso de enseñanza y aprendizaje es la negociación de las representaciones que portan los diversos actores comprometidos en él, puesto que tales representaciones pueden constituirse en facilitadores socio-cognitivos o en una fuente de conflictos. En efecto, la presencia de representaciones sociales en conflicto puede obstaculizar el compromiso de los estudiantes con las tareas de aprendizaje.

En el aprendizaje de una lengua extranjera, son diversas las representaciones sociales que los actores del proceso portan. Estas se refieren, por un lado, a la lengua objeto de aprendizaje, a sus hablantes y a las comunidades que la usan y, por otro, al proceso didáctico propiamente tal, generando expectativas explícitas y subyacentes en torno a su configuración y al rol que le compete asumir a los actores involucrados en él.

De acuerdo con Castellotti y Moore (2002), las representaciones sociales se construyen y reconfiguran en la comunicación, por lo cual son un factor importante de develar en el proceso didáctico de enseñanza de una lengua, puesto que operan potencialmente como facilitadores u obstáculos del proceso. Desde esta perspectiva, es clave interrogarse acerca de cómo se concibe una lengua y el aprendizaje de la misma, particularmente porque los currículos de enseñanza de lengua establecen una serie de temas y procesos que se perciben como compartimientos estancos y sin interconexión entre ellos.

En concreto, Castellotti y Moore (2002) abogan por un enfoque reflexivo de la enseñanza de lenguas extranjeras que incorpore las representaciones sociales de los estudiantes sobre las diferencias culturales y lingüísticas entre su lengua materna y la lengua meta. Dicho enfoque debiera orientarse a la generación de una suerte de descentramiento crítico de la propia lengua y cultura para abrirse a un proceso de razonamiento metacomunicativo. 


\section{Metodología}

El estudio corresponde a un diseño cualitativo de caso único: la experiencia de aprendizaje de una lengua extranjera que poseen estudiantes de la carrera de Lingüística Aplicada a la Traducción de la Universidad de Santiago de Chile, es decir, los estudiantes que se forman como traductores en un contexto monolingüe. El estudio estuvo orientado a generar las condiciones para que estudiantes de $1^{\circ}$ y $5^{\circ}$ años expresaran sus puntos de vista en torno a lo que para ellos significa el dominio de una lengua extranjera, su aprendizaje y enseñanza.

Los sujetos que participaron en el estudio son 14 estudiantes de primer año distribuidos en dos grupos y 9 estudiantes de quinto año distribuidos en dos grupos. El criterio de selección fue la participación voluntaria. Solo se excluyó aquellos estudiantes que hubieran cursado otra carrera universitaria vinculada con el aprendizaje de lenguas. Se utilizaron dos técnicas: una asociación libre de conceptos y una entrevista grupal guiada por una pauta semiestructurada. Ambas técnicas se relacionaron con cuatro tópicos: el dominio de una lengua extranjera, la experiencia de aprendizaje de una lengua extranjera, el proceso de enseñanza de una lengua extranjera y el aprender una lengua extranjera en Chile.

La asociación de conceptos consistió en solicitar a los estudiantes que, por un lapso de un minuto aproximadamente, anotaran individualmente en una hoja todas las palabras y frases que espontánea y rápidamente vincularan con el concepto propuesto por el entrevistador (domino de una lengua extranjera, aprender una lengua extranjera, el proceso de enseñanza de una lengua extranjera y aprender una lengua en Chile). Para el análisis, los investigadores fueron agrupando las palabras y frases de acuerdo con rasgos semánticos compartidos con el fin de construir una categoría más abstracta. Por ejemplo, el concepto de dominio de una lengua extranjera, los entrevistados lo asociaron con "viajar" (4 menciones), "otros países" (2 menciones), "conocer países" (2 menciones). El rasgo semántico compartido en estos términos se caracterizó como "desplazamiento geográfico". Asimismo, el mismo concepto se asoció con "conocimiento cultural" (3 menciones), "cultura" (3 menciones), "política" (1 mención) y “otra 
cultura" (1 mención). El rasgo semántico compartido, se caracterizó como "desplazamiento sociocultural".

Las entrevistas fueron grabadas previo consentimiento por parte de los participantes. Una vez transcritas, se deliberó un proceso familiarización con el contenido que expresaban mediante la lectura reiterada. Dicho proceso, facilitó la segmentación temática de cada entrevista y un proceso de comparación constante con el fin de identificar similitudes y diferencias en las representaciones expresadas por los cuatro grupos de estudiantes.

Posteriormente, considerando las dos fuentes de información y el proceso de comparación entre el discurso de los diferentes grupos, se fue esquematizando la información y se construyeron representaciones visuales que condensarán los elementos fundamentales del discurso de los entrevistados. Dichas representaciones visuales se contrastaron y ajustaron de acuerdo con una relectura de las transcripciones de las entrevistas.

\section{Resultados}

\section{Dominio de una lengua extranjera}

La conceptualización en torno a lo que implica el dominio de una lengua, como lo muestra la Figura 1, difiere entre los estudiantes de primer año y los de quinto año. Los primeros aluden a la interdependencia de tres campos de acción fundamentales: a) el plano de la lengua, b) un sentimiento subjetivo de autoeficacia y c) ampliación de posibilidades de desarrollo personal. Dicha interdependencia, se grafica como un movimiento circular, con el propósito de establecer que "el plano de la lengua" incide en la "sensación subjetiva de autoeficacia y esta, a su vez, incide "en la ampliación de desarrollo personal", lo cual refuerza la seguridad de los sujetos respecto del conocimiento lingüístico y comunicativo en una lengua B o C. En cambio, los estudiantes de quinto año reducen esta conceptualización a dos campos fundamentales que interactúan entre sí: a) el plano de la lengua y b) los aspectos vinculados con la subjetividad del aprendiz (hábitos de estudio, actitud para aprender en términos de compromiso, dedicación y autodisciplina). 


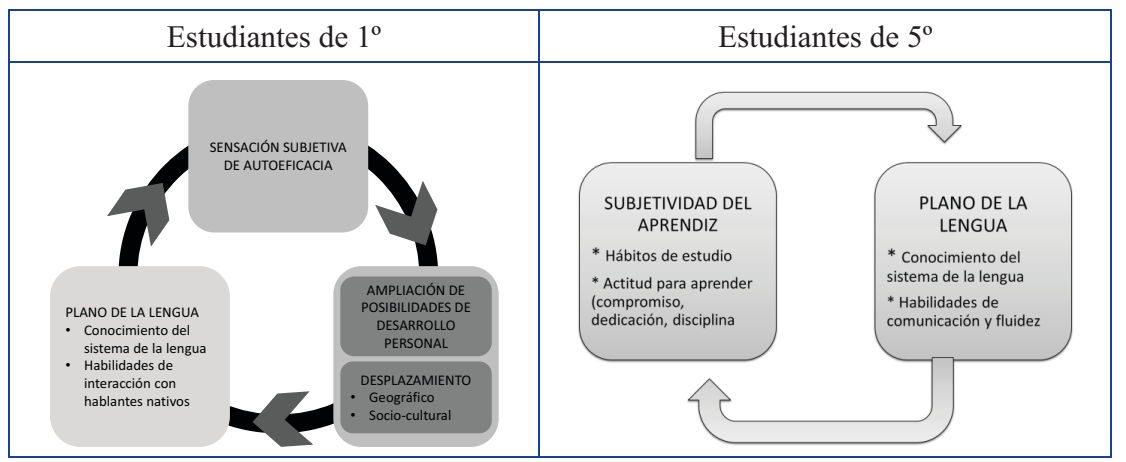

Figura 1 - Diferencias en la conceptualización del concepto dominio de una lengua entre estudiantes de primer y quinto año de traducción.

En otras palabras, la representación sobre el dominio de la lengua va sufriendo una transformación a lo largo del proceso de formación. Si bien el plano de la lengua se mantiene como un campo fundamental en que se evidencia el dominio, su caracterización presenta algunas diferencias entre los estudiantes de los dos niveles. Ambos coinciden en la necesidad de conocer el sistema de las lenguas B o C, pero, en relación a las habilidades comunicativas, los estudiantes de primer año configuran la interacción con hablantes nativos como parámetro fundamental de dominio. La apelación al hablante nativo no aparece en la representación de los estudiantes de $5^{\circ}$ año y es reemplazada por el desarrollo de estrategias metacognitivas para evaluar su propio discurso. Este último grupo de alumnos, posiblemente producto de su formación profesional, tengan mayor conciencia de que la comunidad de hablantes de una lengua se caracteriza por la variación geográfica, social, etaria y estilística. Además, cabe destacar el hecho de que los hablantes efectivos de una lengua tienen diversos niveles de pertenencia e identidad con la comunidad de habla, ya sea porque para algunos constituye su lengua materna, para otros una lengua $\mathrm{B}$ de prestigio en un contexto diglósico y para otros una lengua que favorece el intercambio económico, cultural y científico al adoptarse como una lengua franca.

En relación con la subjetividad, para los estudiantes de primer año esta se significa como una sensación de autoeficacia, un sentimiento de sentirse capaz en el uso efectivo de la lengua. Por el contrario, 
para los estudiantes de $5^{\circ}$ año, dicha subjetividad se manifiesta como un compromiso con un aprendizaje permanente. Posiblemente, los estudiantes de $1^{\circ}$ consideren que el aprendizaje se culmina en un momento dado y los de $5^{\circ}$ hayan adquirido una mayor conciencia de que el desarrollo de la competencia comunicativa en una lengua extranjera implica un proceso de formación continua, el cual demanda una actitud de apertura a nuevos contextos de uso en los cuales siempre es posible seguir aprendiendo.

Es interesante destacar que en el discurso de los estudiantes de $5^{\circ}$ año no hay referencias a la ampliación de posibilidades de desarrollo personal. En este sentido, el desplazamiento geográfico y socio-cultural no se tematiza como un elemento esencial del dominio. Esto no necesariamente implica que no exista como posibilidad o proyección, sino que no se vincula con la conceptualización que se hace de dominio. El núcleo de tal concepto lo constituye el conocimiento y uso de la lengua, el cual se sigue profundizando más allá de la formación profesional, por lo cual demanda una actitud por aprender y hábitos para hacerlo.

\section{El buen aprendiz de lenguas}

Los estudiantes de ambos niveles coinciden en expresar que el compromiso personal es un elemento central en el aprendizaje de una lengua extranjera. Para ellos, el núcleo de dicho compromiso es la motivación y el estudio autogestionado. Su formación se habría gestado en el periodo de la adolescencia, al finalizar la educación básica e iniciar estudios de educación media, momento en el cual el aprender un idioma está directamente vinculado al consumo multimodal, entendido como la interacción con textos multimodales que son de interés para un alumno, de manera que cumple un rol de motivación y medio para el aprendizaje de la lengua en que fueron producidos, tales como música, películas, series, cómics, etc. (Singer et al. 2018). En este caso, el espacio privilegiado de realización de dicho consumo ocurre en internet. Un factor que refuerza dicha motivación estaría constituido por las experiencias de éxito en el espacio escolar. En el discurso de los estudiantes de los dos niveles, el compromiso personal y las experiencias de éxito escolar se retroalimentan mutuamente. 
En el discurso de los entrevistados, la interacción entre compromiso personal y el consumo multimodal es el factor clave que impulsa al aprendizaje sistemático de una lengua extranjera y, además, dinamiza el proceso de adquisición del conocimiento y uso de la lengua. No obstante, existen algunas diferencias, las cuales pueden observarse en la Figura 2.

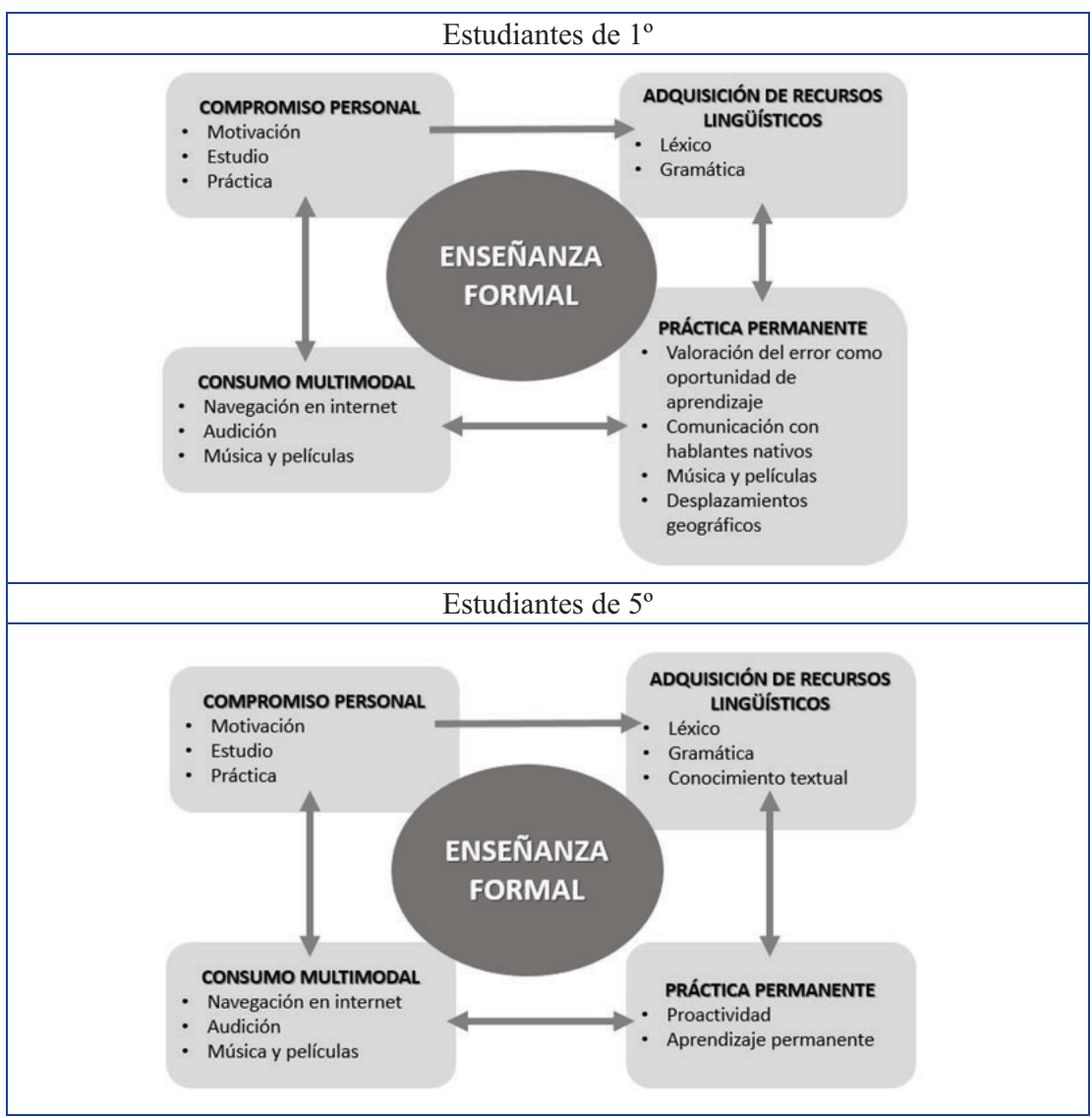

Figura 2 - Comparación de la representación sobre un buen aprendiz de lengua que expresan los estudiantes de primero y quinto años de traducción

En el caso de los estudiantes de ambos niveles, el "compromiso personal" es un factor clave en la "adquisición de recursos lingüísticos" (explicado con una flecha unidireccional en la figura). Ahora bien, el "compromiso personal" interactúa con el "consumo multimodal" 
(interacción representada con la flecha bidireccional) y "la adquisición de recursos lingüísticos" interactúa con la "práctica permanente" de las lenguas B o C (interacción representada con la flecha bidireccional). En gran medida, dicha "práctica permanente" se refuerza autónomamente mediante la interacción con el "consumo multimodal" que, según el discurso de los entrevistados, ellos realizan. No obstante, en la adquisición de los recursos lingüísticos, los estudiantes de $5^{\circ}$ agregan el conocimiento textual al conocimiento de la lengua, aspecto que no aparece expresado en el discurso de los estudiantes de primer año. Por su parte, en lo referente a la práctica permanente, los estudiantes de $5^{\circ}$ enfatizan una actitud proactiva y la actitud de aprendizaje permanente como aspectos claves para asegurar la combinación de espacios formales con espacios no-formales de uso de la lengua que aprenden. Por su parte, los estudiantes de primer año, nuevamente apelan a la interacción con hablantes nativos y la necesidad de oportunidades de desplazamiento geográfico como partes fundamentales para el aprendizaje de la lengua. En otras palabras, el contar con dichas posibilidades los situaría en la percepción de ser buenos aprendices; el carecer de estas oportunidades implicaría una limitación en el proceso de adquisición y uso de la lengua. Otro aspecto importante que agregan, y que no aparece en el discurso de los de $5^{\circ}$, es la valoración del error como parte del proceso de aprendizaje. Es posible que visualicen más el error, puesto que, en general, su aprendizaje ha sido autogestionado en internet y carecen de un parámetro de corrección externa que valide dicho aprendizaje. En esta línea, los estudiantes de primer año expresan una percepción ambivalente del dominio logrado, cuya valoración negativa se expresa fundamentalmente en la posibilidad de una inadecuación contextual de la selección léxica y en la calidad de la producción oral.

\section{Aprendizaje de una lengua extranjera}

Un aspecto relevante en el aprendizaje de la lengua se relaciona con la percepción del dominio logrado. En este aspecto, es factible reconocer importantes diferencias entre los estudiantes de $1^{\circ}$ y los de $5^{\circ}$, las cuales se expresan en la Figura 3. 


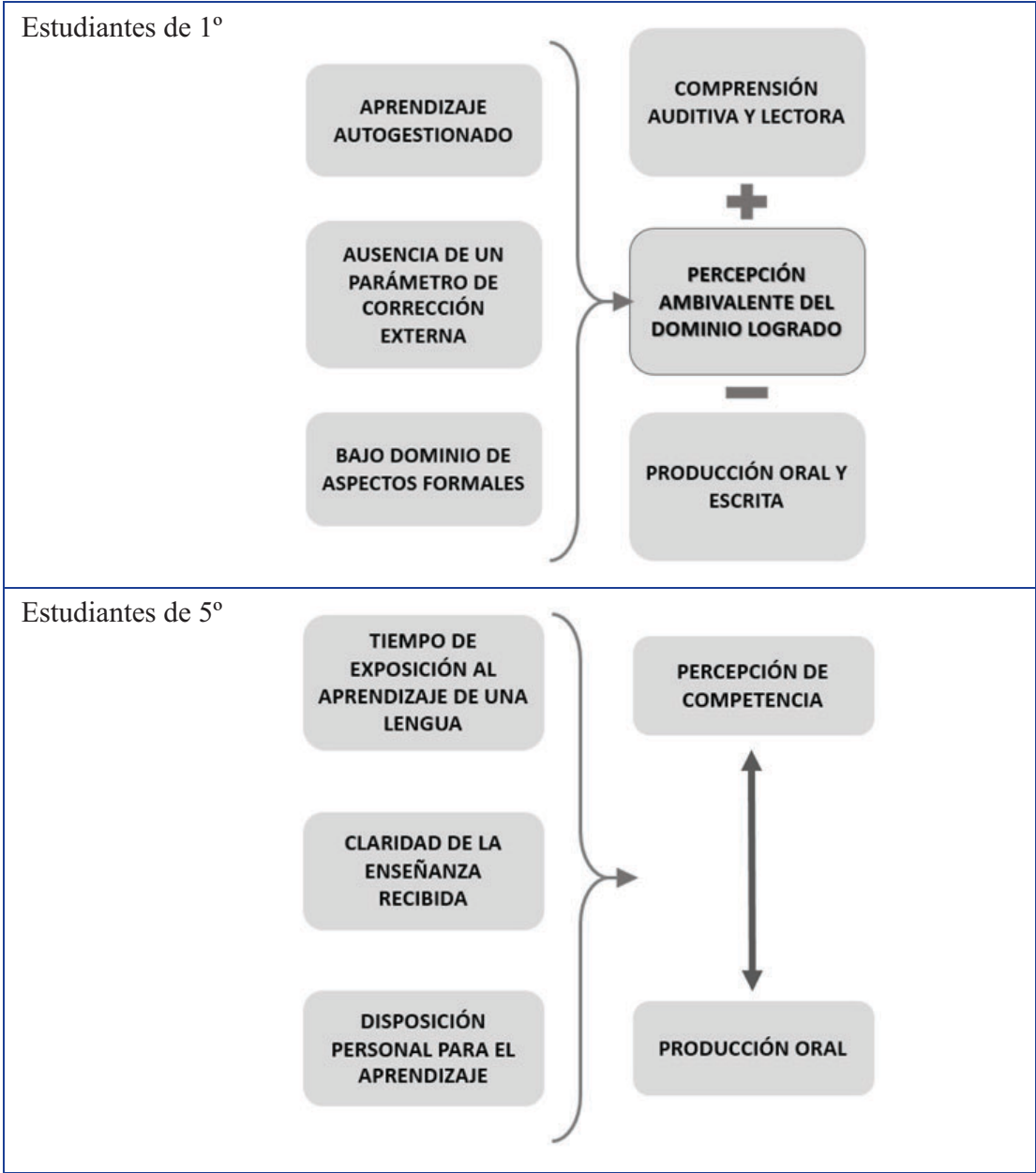

Figura 3 - Comparación del proceso de aprendizaje de una lengua que expresan estudiantes de $1^{\circ}$ y $5^{\circ}$ año de traducción.

Los estudiantes de $1^{\circ}$ acentúan la falta de un espacio formal de aprendizaje, pues este proporcionaría un parámetro de corrección externa que contribuye al mejoramiento de los aspectos formales. En este sentido, su experiencia de aprendizaje autogestionado habría significado una percepción positiva sobre el desarrollo de habilidades de recepción, pues ellos constatan un crecimiento progresivo de su repertorio léxico y su capacidad de construir significados a partir de emisiones orales y escritas. No obstante, sus habilidades de producción son significadas negativamente. Tanto en la producción oral como en la 
escrita evidencian una mayor inseguridad e identifican obstáculos para una comunicación fluida, precisa y contextualmente adecuada.

Por el contrario, los estudiantes de $5^{\circ}$ expresan una percepción de mayor competencia, la cual está condicionada por el tiempo de exposición al aprendizaje de una lengua, la calidad de la enseñanza recibida y la disposición personal para el aprendizaje. Esta percepción se valida fundamentalmente por su desempeño en habilidades de producción oral. Sería este el parámetro fundamental desde el cual se evalúa un buen manejo de la lengua aprendida.

Me siento más competente en portugués, porque siento que puedo hablar mejor en portugués que en inglés. En inglés me puedo manejar como escribiendo y comprensión de textos, pero hablando siento que todavía no soy capaz de hablar bien y de darme a entender bien en inglés (A1, G5-1).

En otras palabras, la percepción de competencia se relaciona con la posibilidad de producción espontánea, fundamentalmente oral. La posibilidad de mantener una conversación con otros se constituye en el parámetro fundamental de dominio. Dicha capacidad se caracteriza como la posibilidad de construcción de un marco de entendimiento mutuo que facilite la interacción comunicativa. En este sentido, la percepción de competencia no se relaciona con el logro de un buen desempeño académico, sino con un aspecto subjetivo relevante: la capacidad de hablar, de saber usar la lengua en un contexto de interacción.

Al mismo tiempo, para los estudiantes de $5^{\circ}$, la percepción de competencia interactúa con la familiarización con la cultura de la lengua meta, aspecto clave desde el cual juzgan la adecuación discursiva. En consecuencia, según su propio discurso, estos dos aspectos capacitan al estudiante para reponerse a prácticas docentes calificadas como de baja calidad.

Un aspecto importante, tanto en los estudiantes de $1^{\circ}$ como de $5^{\circ}$, es contar con una retroalimentación explícita del desempeño por un sujeto que tenga mejor dominio de la lengua: "yo quiero que me enseñen, porque yo sé que puedo estudiar sola, pero quiero que me enseñen, quiero que me corrijan" (A1, G5-2). En esta línea, un buen proceso de aprendizaje implica la vivencia de un evento comunicativo estructurado y caracterizado por un clima de confianza que posibilite la corrección empática por parte del profesor. 


\section{Enseñanza de una lengua extranjera}

Un primer aspecto que los entrevistados destacan cuando se refieren a la enseñanza de una lengua extranjera se refiere al rol del docente. $\mathrm{Al}$ expresar la visión que se tiene sobre dicho rol, hay dominios relativamente comunes para definir dicha labor. No obstante, al caracterizar dichos dominios es posible evidenciar algunas diferencias entre los estudiantes de $1^{\circ}$ y los de $5^{\circ}$ año, lo que puede apreciarse en la Figura 4.

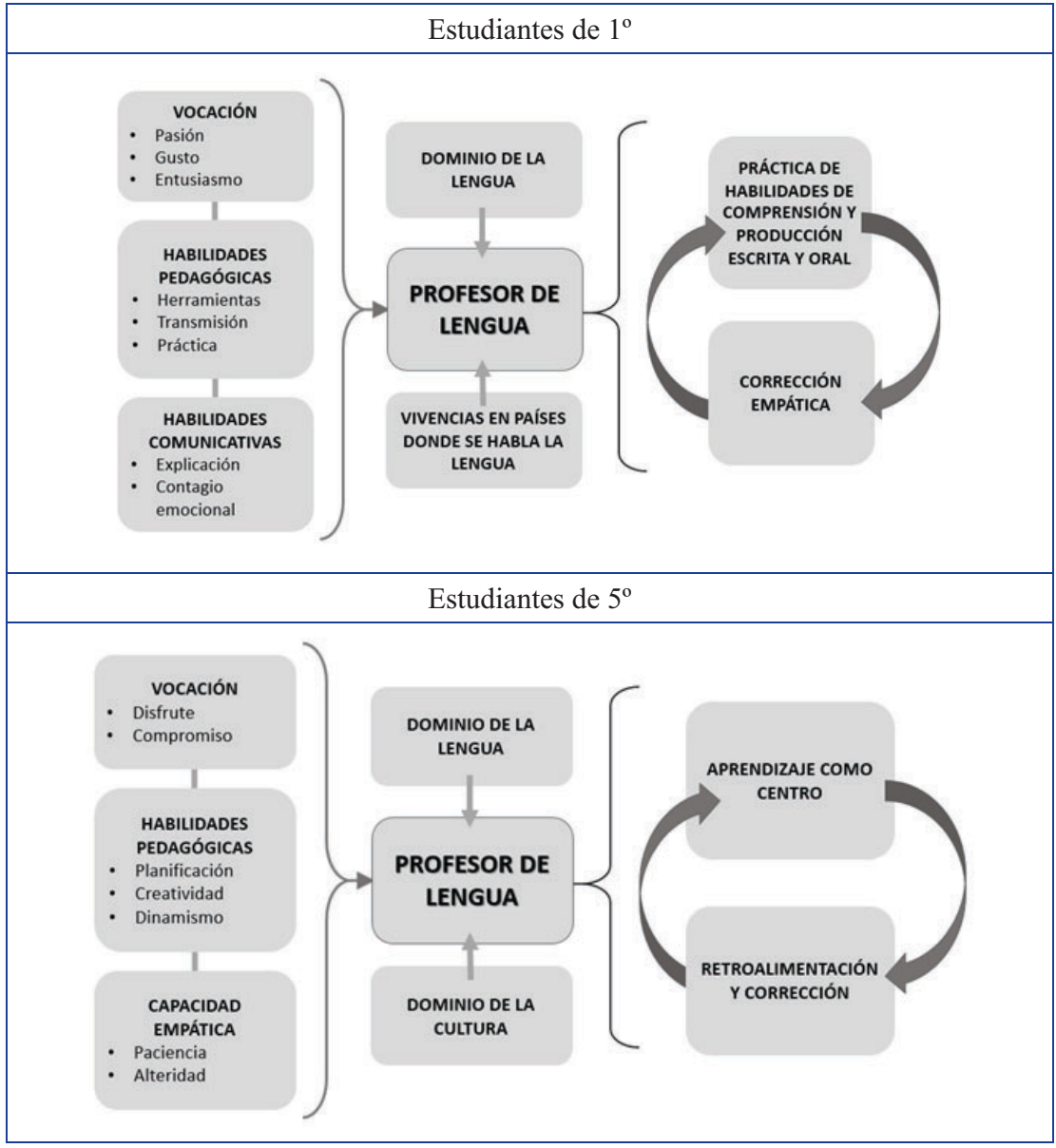

Figura 4 - Comparación de la representación sobre la labor de un buen profesor de lengua que manifiestan estudiantes de $1^{\circ}$ y $5^{\circ}$ año. 
Respecto de la vocación como condición fundamental para definir a un buen profesor de lengua, los grupos tienden a coincidir. En la descripción de las habilidades pedagógicas, los estudiantes de $1^{\circ}$ evidencian una visión de la enseñanza centrada en la transmisión de contenidos. Desde esta perspectiva, el profesor debe poseer herramientas que faciliten una buena presentación de los contenidos y promover, posteriormente, la práctica de los mismos. Los estudiantes de $5^{\circ}$, enfatizan más claramente la labor antes y durante la clase orientada a estimular el compromiso de los estudiantes con el aprendizaje. En este sentido, un buen profesor estructura la clase como evento comunicativo, en que los aspectos interactivos se ponen en relieve, interpelando así la participación. Congruentemente, para los estudiantes de $1^{\circ}$, las habilidades comunicativas del docente se significan en la perspectiva de la explicación y el contagio emocional. Pareciera que el éxito de la clase está en directa relación con el quehacer del docente, evidenciándose cierta pasividad por parte de los estudiantes. Para los estudiantes de $5^{\circ}$ dichas habilidades se conceptualizan como capacidad empática, es decir, la capacidad del profesor de ponerse en el lugar de los estudiantes presentándole estímulos que respondan a diferentes estilos de aprendizaje.

Los estudiantes de ambos niveles coinciden en que el profesor debe tener un dominio de la lengua meta y un conocimiento de la cultura de los países en que se habla. No obstante, para los estudiantes de $1^{\circ}$ dicho conocimiento implica haber vivido en un país en que se hable la lengua. Dicho desplazamiento geográfico y socio-cultural los validaría como buenos profesores de lengua, pues estarían transmitiendo un conocimiento de carácter experiencial. Este aspecto no aparece en el discurso de los estudiantes de $5^{\circ}$.

Respecto de la enseñanza de la lengua, los estudiantes de $1^{\circ}$ enfatizan la práctica de las macrohabilidades comunicativas (escuchar, leer, hablar y escribir) y la corrección por parte del profesor. En este sentido, la ejercitación del contenido expuesto requiere contar con un parámetro externo de corrección. Este es un elemento que articula su discurso sobre un buen profesor, aunque no describen como se pone en práctica. Para los estudiantes de $5^{\circ}$, ese parámetro sigue siendo relevante, pero lo complementan con la idea de la retroalimentación. En esta línea, se oponen a una clase centrada solo en la transmisión de 
contenidos, sino que ponen el acento en que un buen profesor se orienta a conocer el nivel de manejo que los estudiantes tienen para proyectar una progresión en el aprendizaje, es decir, es el progreso concreto de los estudiantes el parámetro en torno al cual planificar y estructurar los medios de ayuda. Esto significa que el profesor debe manejar un buen conocimiento de los intereses, motivaciones y objetivos de los estudiantes con los que trabaja; utilizar el tiempo en forma eficiente; diseñar materiales interesantes y variados, evitando caer en la rutina; favorecer el uso de lo aprendido en situaciones cotidianas, estimulando la conversación espontánea; incorporar el abordaje de técnicas de estudios para potenciar el compromiso de sus estudiantes con el aprendizaje.

Una mala clase es cuando los ramos de... como de progresión, por ejemplo de inglés, pasamos, terminamos inglés y después vienen como redacción, esos ramos que consideran los contenidos que se supone que aprendimos antes, no están al nivel de lo que alcanzamos antes. Como que el profesor, no... su planificación no está acorde al nivel que se suponen que alcanzaron los estudiantes para llegar a ese ramo y siento que no estoy aprendiendo nada (A1, G5-2).

Como se ve en la cita, los estudiantes de $5^{\circ}$ tienen una mayor sensibilidad a la articulación que hay entre un nivel y otro, así como la coordinación que debiera haber entre los ramos de un mismo nivel para evitar la reiteración innecesaria y ajustar la debida complementariedad entre asignaturas. Otro aspecto sensible que estos estudiantes destacan es el ritmo de enseñanza, el cual debe estar dosificado desde la perspectiva de las capacidades de los estudiantes, la estructuración de la ayuda pedagógica y la interacción comunicativa.

En japonés como que el profesor tenía como una meta: "ya, en la semana hay que pasar dos lecciones por semana" e iba como avión y yo no entendía nada porque iba súper rápido y solamente teníamos que alcanzar esa meta. Entonces, como que la meta nunca era que aprendiéramos (A3, G5-2).

Lo otro que me molesta a mí son los profes, sobre todo en el área de japonés, que ocupan textos que están desactualizados. No preparan la clase, llegan a leer la cuestión a la sala y eso a mí me molesta porque el lenguaje, o sea, la lengua va evolucionando con la cultura, o sea, de qué estamos hablando si no están actualizados en eso, o sea, ¿qué nivel vamos a tener nosotros? ¿De qué 
estamos hablando? Si un profe no se impregna de la cultura, ¿cómo contagiar eso a los alumnos? ... Me gustaría que fuera diferente, porque siento que hay muchas cosas que se pueden hacer, siendo que acá hay secciones chicas, de poquitos alumnos y que se pueden hacer cosas muy buenas y hay profes que sí lo saben aprovechar. No voy a decir que no, pero hay varios que no, que lo desaprovechan mucho, sobre todo en ramos que son, por ejemplo, no sé po', fonética, que se podría hacer súper didáctico. A eso me refiero y eso es lo que le falta a la carrera, como más enfoque práctico. Yo creo que todas estamos de acuerdo en que nosotras sabemos cómo escribir perfecto así o revisar textos, corregir y todo, pero al momento de hablar, a lo mejor, no tenemos tanta creatividad o tanto desplante por lo mismo, porque se enfocan mucho en aprender teoría, estructura al revés y al derecho, pero dejan mucho de lado la parte como personal y libre que tienen los idiomas po'. Si al final uno empieza a pensar en el idioma, tiene que saber demostrarlo también (A2, G5-2).

Pareciera que los estudiantes de $5^{\circ}$ apelan a una clase que combina lo estructurado con la acogida de situaciones emergentes, lo que posibilita el uso espontáneo de la lengua.

Finalmente, los entrevistados de ambos niveles destacan las dificultades que implica aprender una lengua extranjera en un contexto monolingüe, destacando el costo, la subvaloración y la falta de espacios para una suerte de uso auténtico de la lengua. De todos modos, ambos grupos destacan que es posible hacerlo, siempre y cuando el aprendiz se comprometa y persevere.

\section{Conclusiones}

Este estudio evidencia que las representaciones sociales de los alumnos, en efecto, pueden mantenerse, reforzarse, cambiar o desaparecer a lo largo de la trayectoria formativa. En este sentido, se pueden observar algunos hitos importantes en cada una de las dimensiones analizadas.

En relación al dominio de una lengua extranjera, se evidencia la desaparición del hablante nativo como facilitador y referente lingüístico sostenido en primer año y es reemplazado por estrategias metacognitivas para evaluar su progreso y monitorear el nivel de su producción. Por 
otra parte, en el ámbito de la subjetividad, los alumnos de primer año conciben la competencia comunicativa como un producto, mientras que los de quinto año lo entiende como un proceso en constante desarrollo. Más aún, se evidencia una actitud de constante perfeccionamiento en el uso y conocimiento de la lengua que reemplaza el desplazamiento físico y socio-cultural.

En la segunda dimensión, relacionada con el buen aprendiz de una lengua extranjera, las representaciones en torno al buen alumno convergen en una serie de elementos: el aprendizaje autogestionado y la motivación. Este último factor se nutre del nexo entre compromiso personal y el consumo multimodal. La combinación resultante pareciera potenciar los procesos de enseñanza-aprendizaje. No obstante, mientras que los alumnos de quinto apuntan a una actitud proactiva en el aprendizaje, los alumnos de primer año enfatizan factores situacionales y contextuales como agentes que potencian a un buen alumno. Finalmente, se evidencia la ausencia de la concepción del error como instancia de aprendizaje en el discurso de los alumnos de quinto año.

En la tercera dimensión, el aprendizaje de una lengua extranjera, se observan posturas diferentes. Por una parte, los alumnos de primer año enfatizan la necesidad de un espacio formal de aprendizaje, ya que su aprendizaje autogestionado no permite la posibilidad de retroalimentación sistemática. En consecuencia, presentan aprehensiones en cuanto a su capacidad de producción oral y escrita. Por otra parte, los alumnos de quinto presentan una visión más precisa, donde identifican el tiempo de exposición, calidad de la enseñanza y disposición a aprender. De esta forma, se puede establecer que la competencia comunicativa oral es el parámetro fundamental que los estudiantes entrevistados identifican de dominio de la lengua extranjera. Para esto, ambos niveles establecen que es necesaria una retroalimentación sistemática durante el proceso de aprendizaje.

Por último, en cuanto a la enseñanza de segundas lenguas, se evidencia visiones diferentes en relación al rol del profesor. Los alumnos de primer año perfilan al docente desde un paradigma más tradicional en que este presenta contenidos y luego otorga oportunidades de práctica. Los alumnos de quinto construyen sobre estas representaciones y elaboran conceptos más complejos asociados a la práctica significativa 
que potencie la competencia comunicativa. Asimismo, ambos apuntan a la capacidad empática del profesor durante el proceso de enseñanza, así como el conocimiento pedagógico. No obstante, los alumnos de primer año suponen que dicho conocimiento requiere que el docente haya vivido en un país donde la lengua estudiada es la lengua oficial, mientras que esto desaparece en el discurso de los alumnos de quinto año.

Además, los alumnos de quinto señalan que la clase de lengua debería trascender el mero traspaso de conocimientos. Según ellos, el buen profesor de lengua debe ser capaz de organizar el contenido de manera coherente y significativa. Para esto, resulta fundamental que el profesor sea organizado, esté familiarizado con los intereses y motivaciones de sus alumnos para potenciar técnicas de estudio de acuerdo a sus aptitudes. En este sentido, probablemente debido a su exposición en el programa por, a lo menos, 5 años, son capaces de identificar problemas de adaptación curriculares y a nivel de programa y de asignaturas. De esta forma, también enfatizan los efectos negativos que esto puede tener en la motivación de los alumnos y, finalmente, en su capacidad de producción oral.

Se concluye que las representaciones de los alumnos presentan cambios en las dimensiones estudiadas, relativas al aprendizaje y enseñanza de lenguas extranjeras, como resultado de las experiencias dentro de la carrera de Lingüística Aplicada a la Traducción. En concreto, la disposición de pasar desde un aprendizaje guiado hacia uno donde los alumnos son capaces de desarrollar estrategias de aprendizaje y evaluarlas metacognitivamente permite establecer que la adopción de metodologías más activas como la de Beeby (2004) o Bernardini $(2004,2016)$ son posible, al menos desde la perspectiva de los estudiantes. Futuros trabajos relacionados con esta materia podrían explorar las representaciones de los docentes en torno a los lineamientos pedagógicos que subyacen estas propuestas metodológicas.

En cuanto a las posibles limitaciones de este trabajo, se consideran las representaciones correspondientes al primer y quinto año, pero no se evidencia la forma en que las representaciones evolucionaron en los diferentes niveles de la carrera de Lingüística Aplicada a la Traducción. En este sentido, una posible proyección de este trabajo involucraría un estudio longitudinal que evidencie el cambio paulatino cognitivo del alumno de traducción a lo largo del proceso de formación, así como el 
impacto de estas representaciones en la configuración de la identidad profesional traductora. A la fecha de redacción de este artículo, este aspecto no se considera como factor relevante para definir el éxito de una propuesta didáctica para la enseñanza de lenguas en estudios de traducción, por lo que estudios sobre estos temas contribuirían de manera sustancial al debate y propuestas metodológicas para la enseñanza de lenguas en la formación profesional de traductores en contextos monolingües, particularmente en Chile y Latinoamérica.

\section{Referencias bibliográficas}

AGOST, Rosa. 2008. Enseñar la teoría de la traducción: diseño de competencias y explotación de recursos pedagógicos. Quaderns. Revista de Traducció, 15: 137-152.

BEEBY, Allison. 2004. Language learning for translators: Designing a syllabus. En: Kristen Malmkjær (ed.). Translation in undergraduate degree programmes. Amsterdam: John Benjamins, p. 39-65.

BERENGUER, Laura. 1996. Didáctica de segundas lenguas en los estudios de traducción. En: Amparo Hurtado Albir. Ed. La enseñanza de la traducción. Castelló: Publicacions de la Universitat Jaume I, p. 9-30.

BERNARDINI, Silvia. 2000. Competence, capacity, corpora - A study in corpus-aided language learning. Bologna: Clueb.

BERNARDINI, Silvia. 2004a. The theory behind the practice: Translator training or translator education? En: Kristen Malmkjær. Ed. Translation in undergraduate degree programmes. Amsterdam: John Benjamins, p. 17-30.

BERNARDINI, Silvia. 2004b. Corpus-aided language pedagogy for translator education. En: Kristen Malmkjær. Ed. Translation in undergraduate degree programmes. Amsterdam: John Benjamins, p. 97-111.

BERNARDINI, Silvia. 2016. Discovery learning in the languagefor-translation classroom: Corpora as learning aids. Cadernos de Traduçao, 36/especial, p. 14-35.

CALVO, Elisa. 2017. Servicios de valor añadido en contextos situacionales en traducción: De los proyectos al portafolio. RIDU, 11/2, p. 136-154.

CARVAJAL, Romina Valeria. 2017. Propuesta didáctica para abordar la traducción técnica: Trabajo colaborativo y aprendizaje basado en proyectos. RIDU, 11/2, p. 192-202. 
CHAIB, Mohamed. 2015. Social representations, subjectivity and learning. Cadernos de pesquisa, 45/156, p. 359-371.

CASTELLOTTI, Véronique \& Danièle Moore. 2002. Social representations of languages and teaching. Strasbourg: Council of Europe.

CORTÉZ, José. 2009. Nuevas metodologías en enseñanza de la traducción: Una perspectiva cognitiva. Mutatis Mutandis, 2/2, p. 282-294.

CRUZ GARCÍA, Laura. 2017. Enseñanza de lenguas extranjeras en facultades de traducción e interpretación españolas: Estudio de factores textuales. $R I D U, \mathbf{1 1} / \mathbf{2}$, p. 75-89.

DELISLE, Jean. 1980. L'Analyse du discours comme méthode de traduction: initiation à la traduction française de textes pragmatiques anglais, théorie et pratique. Ottawa: Presses de l'Université d'Ottawa.

HANSEN, Gyde. 1997. Success in translation. Perspectives: Studies in Translatology, 5/2, p. 201-210.

HEWSON, Lance \& Jacky Martin. 1991. Redefining translation. The variational approach. London/New York: Routledge.

HOLMES, James S. 1972. The name and nature of translation studies. En: James Holmes Translated! Papers on literary translation and translation studies. Amsterdam: Rodopi, p. 67-80.

HURTADO ALBIR, Amparo. 1996a. La enseñanza de la traducción directa 'general'. Objetivos de aprendizaje y metodología. En: Amparo Hurtado Albir. Ed. La enseñanza de la traducción. Castellón: Universitat Jaume I, p. 31-55.

HURTADO ALBIR, Amparo. 1996b. La cuestión del método traductor. Método, estrategia y técnica de traducción. Sendebar, 7, p. 39-57.

HURTADO ALBIR, Amparo. 1999. enseñar a traducir. Madrid: Edelsa.

HURTADO ALBIR, Amparo. 2017. Researching translator competence by PACTE Group. Amsterdam/Philadelphia: John Benjamins.

JODELET, Denise. 1988. La representación social: fenómenos, conceptos y teoría. En: Sege Moscovici. Ed. Psicología social II. Pensamiento y vida social, Barcelona: Paidós, p. 469-494.

JODELET, Denise. 2008. El movimiento de retorno al sujeto y el enfoque de las representaciones sociales. Cultura y representaciones sociales, 5, p. 32-63.

KELLY, Dorothy. 2005. A handbook for translator trainers. Oxford: St Jeronme Publishing.

KIRALY, Donald. 1995. Pathways to translation. Pedagogy and process. Kent, OH: The Kent State University Press.

KIRALY, Donald. 2000. A social constructivist approach to translator education. Manchester: St Jeronme Publishing.

KORNBLIT, Ana Lía. 2007. Metodologías cualitativas en ciencias sociales. Modelos y procedimientos de análisis. Buenos Aires: Biblos. 
MORALES, Ángela. 2017. Diseño de un proyecto de traducción para desarrollar la competencia temática. RIDU, 11/2, p. 170-191.

MOSCOVICI, Serge. 1979. El psicoanálisis, su imagen y su público. Buenos Aires: Huemul.

NEUBERT, Albrecht. 1994. Competence in translation: a complex skill, how to study and how to teach it. En: Marry Snell-Hornby, Franz Pöchhacker \& Klaus Kaindl (eds.). Translation studies. An interdiscipline. Amsterdam/Philadelphia: John Benjamins, p. 411-420.

NORD, Christiane. 1991. Text analysis in translation. Amsterdam: Rodopi.

NORD, Christiane. 1992. Text analysis in translator training. En: Cay Dollerup \& Annette Lindegaard. Eds. Teaching translation and interpreting 1. Amsterdam/Philadelphia: John Benjamins, p. 39-48.

PACTE. 2017. PACTE translation competence model. A holistic, dynamic model of translation competence. En: Amparo Hurtado Albir. Ed. Researching translator competence by PACTE Group. Amsterdam/ Philadelphia: John Benjamins, p. 35-41.

RISKU, Hanna. 1998. Translatorische Kompetenz. Kognitive Grundlagen des Übersetzens als Expertentätigkeit.Tübingen: Stauffenburg.

ROBERTS, Roda P. 1984. Compétence du nouveau diplômé en traduction. En: Conseil de la langue française Traduction et Qualité de Langue. Actes du Colloque Société des traducteurs du Québec. Québec: Éditeur officiel du Québec, p. 172-184.

SINGER, Néstor. 2016. A proposal for language teaching in translator training programmes using data-driven learning in a task-based approach.International Journal of English and Translation Studies, 4/2, p. 155-167.

SINGER, Néstor, Manuel Rubio \& Raquel Rubio. 2018. Representaciones de estudiantes de traducción en el aprendizaje de una lengua extranjera, Onomázein, 39, p. 245-269. [DOI 10.7764/onomazein.39.10].

WILSS, Wolfram. 1976. Perspectives and limitations of a didactic framework for the teaching of translation. En: Richard W. Brislin. Ed. Translation Applications and Research. New York: Gardner, p. 117-137.

WILSS, Wolfram. 2004. Translation studies: A didactic approach. En: Kristen Malmkjær. Ed. Translation in undergraduate degree programmes. Amsterdam: John Benjamins, p. 9-16.

Recebido em: 28/06/2018

Aprovado em: 22/08/2019 